\title{
In vivo variability in quantitative coronary ultrasound and tissue characterization measurements with mechanical and phased-array catheters
}

\author{
Gastón A. Rodriguez-Granillo, Eugène P. Mc Fadden, Jiro Aoki, \\ Carlos A. G. van Mieghem, Evelyn Regar, Nico Bruining \& Patrick W. Serruys \\ Thoraxcenter, Erasmus Medical Center, Bd406, Dr. Molewaterplein 40, 3015 GD Rotterdam, \\ The Netherlands
}

Received 3 March 2005; accepted in revised form 26 April 2005

Key words: atherosclerosis, imaging, plaque characterization, ultrasonography

\begin{abstract}
Background: Both mechanical and phased-array catheters are used in clinical trials to assess quantitative parameters. Only limited evaluation of the in vivo agreement of volumetrical measurements between such systems has been performed, despite the fact that such information is essential for the conduction of atherosclerosis regression trials. Methods and results: We prospectively evaluated the agreement in morphometric measurements and intravascular ultrasound (IVUS)-based plaque characterization between a $40 \mathrm{MHz}$ rotating transducer (3.2 F Atlantis, Boston Scientific Corp.) and a $20 \mathrm{MHz}$ phased-array catheter (2.9 F Eagle Eye, Volcano Therapeutics, Rancho Cordova, California) in 16 patients. Lumen $\left(7.3 \pm 2.0 \mathrm{~mm}^{2}\right.$ vs. $\left.6.7 \pm 1.8 \mathrm{~mm}^{2}, p=0.001\right)$ and vessel $\left(11.8 \pm 3.3 \mathrm{~mm}^{2}\right.$ vs. $11.0 \pm 2.9 \mathrm{~mm}^{2}$, $p=0.02$ ) cross-sectional areas (CSA) were significantly greater with the $20 \mathrm{MHz}$ system. Plaque CSA measurements showed no significant difference between systems $\left(4.4 \pm 2.3 \mathrm{~mm}^{2}\right.$ vs. $\left.4.4 \pm 2.1\right)$. The relative differences were less than $10 \%$ for the three variables. On IVUS-based tissue characterization (13 patients), calculated percentage hypoechogenic volume was significantly higher for the $20 \mathrm{MHz}$ system $(96.7 \pm 2.38$ vs. $88.4 \pm 5.53, p<0.0001)$. Conclusions: Quantitative IVUS analyses display significant catheter type-dependent variability. It is unclear whether the variability reflects overestimation of measurements with the phased-array or underestimation with the mechanical system. Although plaque burden measurements did not differ significantly between systems, it appears prudent to recommend the use of a single system for progression/regression studies.
\end{abstract}

\section{Introduction}

Intravascular ultrasound (IVUS) allows a high resolution tomographic assessment of the coronary artery and provides accurate measurements of both lumen and vessel wall dimensions. Initially used in interventional cardiology for diagnostic and interventional procedures, IVUS has more recently been used as a tool to assess atherosclerosis progression/regression in single and multicenter studies, given its ability to accurately quantify the presence and extent of plaque formation [1-3]. In addition, plaque characterization using gray-scale IVUS and the spectral analysis of the raw radiofrequency data is subject to intensive research [4-6]. Currently, a number of IVUS systems are commercially available and there is limited in vivo data regarding the agreement between mechanical and phased-array catheters although this information is valuable 
for the conductance of multicenter progression/ regression studies. Previous in vitro and in vivo data showed significant variability between different catheters in quantitative and tissue characterization data [7-9]. The purpose of this study was to compare in vivo the quantitative coronary ultrasound measurements and plaque characterization with mechanical and phased-array catheters.

\section{Materials and methods}

\section{Patient population}

Patients were eligible if they had a de novo, nonsignificant (angiographically $<50 \%$ ) stenosis in a native coronary artery. Patients were excluded from the study if any of the following conditions were present: (1) presentation with acute coronary syndrome, (2) vessel tortuosity (3) calcified vessels. The institutional ethics committee approved the study protocol and written informed consent was obtained from all patients.

\section{IVUS imaging systems}

Two commercially available systems were used: a single-element, $40 \mathrm{MHz}$ rotating transducer (3.2 F Atlantis, Boston Scientific Corp.), and a $20 \mathrm{MHz}$ phased-array catheter (2.9 F Eagle Eye, Volcano Therapeutics, Rancho Cordova, California).

\section{Vessel interrogation}

IVUS was performed after intracoronary administration of nitrates. Cine runs, before and during contrast injection, were performed to define the position of the IVUS catheter $\geq 10 \mathrm{~mm}$ distal to a clear anatomical landmark. Using an automated pullback device, the transducer of the phased array catheter was withdrawn at a continuous speed of $0.5 \mathrm{~mm} / \mathrm{s}$ until the ostium of the study vessel was seen. Subsequently, the same procedure was performed with the other IVUS imaging catheter using a different automated pullback device (Boston Scientific Corp, Santa Clara, USA) at the same speed. IVUS data was stored on S-VHS videotape. The videotapes were digitized on a computer system, transformed into the DICOM medical image standard and stored on an IVUS Picture Archiving and Communications System (PACS).

\section{IVUS analysis}

Quantitative coronary ultrasound (QCU) analysis was performed by a core laboratory (Cardialysis BV, Rotterdam, The Netherlands) using validated software (Curad, version 3.1, Wijk bij Duurstede, The Netherlands). IVUS analysts were not aware of the purpose of the study. The regions of interest (ROI) were matched simultaneously for the two systems and selected by an independent observer who did not participate in the contour detection and subsequent analysis. The borders of the external elastic membrane (EEM) and the lumenintima interface were determined with manual planimetry and enclosed a volume that was defined as the coronary plaque plus media volume. Lumen (LCSA), vessel (VCSA), and plaque (PCSA) cross sectional areas (CSA) were evaluated. Plaque CSA was calculated as:

$$
\text { PCSA }=\text { Vessel }_{\text {area }}-\text { Lumen }_{\text {area }}
$$

\section{IVUS tissue characterization}

In addition to volumetric parameters, IVUS also provides information on plaque echogenicity, a potential source of information on plaque composition. The acoustic characterization of a coronary plaque has been investigated by in vitro and in vivo studies that support a role for echogenicity as a predictor of histological plaque composition $[1,6,10-12]$. In the present study, we used a computer-aided grey scale value analysis program for plaque characterization [13]. Using the mean grey level of the adventitia as a threshold, five main tissue types can be characterized (Figure 3): (1) hypoechogenic tissue has a mean grey level lower than that of the adventitia, (2) hyperechogenic tissue, defined as tissue with a mean grey value higher than that of the adventitia, (3) calcified tissue, defined as a tissue with a mean grey value higher than that of the adventitia with associated acoustic shadowing, (4) unknown 
tissue, defined as tissue shadowed by calcification and (5) 'upper tissue', defined as tissue that has a mean grey value higher than the mean adventitial intensity plus two times its standard deviation but is not typical calcified tissue with acoustic shadowing. The percentage of hypoechogenic plaque was calculated for the entire ROI, excluding 'upper tissue'.

\section{Statistical analysis}

Results are reported as mean \pm standard deviation. Bland-Altman plots were constructed in order to assess the agreement between measurements with both types of catheter [14]. This method plots the mean against the difference in measurements between catheters. Limits of agreement were set by adding two SDs to the mean difference for the upper limit and by substracting two SDs from the mean difference for the lower limit. A $p$ value of less than 0.05 indicated statistical significance.

\section{Results}

Sixteen patients were included in the analysis. The mean age was $64 \pm 9$ years (range 49-82), 9 patients $(56.3 \%)$ were males. The study vessel location was RCA 4 (25\%), LCX $5(31 \%)$ and LAD 7 (44\%). Table 1 shows CSA measurements with the two systems. Lumen $\left(7.3 \pm 2.0 \mathrm{~mm}^{2}\right.$ vs $\left.6.7 \pm 1.8 \mathrm{~mm}^{2}, \quad p=0.001\right) \quad$ and vessel $\left(11.8 \pm 3.3 \mathrm{~mm}^{2}\right.$ vs. $\left.11.0 \pm 2.9 \mathrm{~mm}^{2}, p=0.02\right)$ CSAs were significantly larger with the $20 \mathrm{MHz}$. PCSA measurements showed no significant

Table 1. Cross-sectional area measurements for two different IVUS imaging catheter systems (n:16).

\begin{tabular}{lclcl}
\hline & Length & LCSA & VCSA & PCSA \\
\hline $20 \mathrm{MHz}$ & $37.1 \pm 16.8$ & $7.3 \pm 2.0$ & $11.8 \pm 3.3$ & $4.4 \pm 2.3$ \\
$40 \mathrm{MHz}$ & $35.7 \pm 15.7$ & $6.7 \pm 1.8$ & $11.0 \pm 2.9$ & $4.4 \pm 2.1$ \\
Absolute $\Delta$ & $1.4 \pm 2.2$ & $0.6 \pm 0.7$ & $0.7 \pm 0.9$ & $0.1 \pm 0.4$ \\
Relative $\Delta$ & $3.0 \pm 5.8$ & $9.3 \pm 8.7$ & $5.9 \pm 6.7$ & $-1.4 \pm 13.4$ \\
p value & 0.023 & 0.001 & 0.005 & $\mathrm{NS}$ \\
\hline
\end{tabular}

LCSA, VCSA and PCSA refer to lumen, vessel and plaque cross-sectional areas. difference between systems $\left(4.4 \pm 2.3 \mathrm{~mm}^{2}\right.$ vs $4.4 \pm 2.1, p=\mathrm{NS})$. The relative differences were less than $10 \%$ for the 3 variables. Bland-Altman plots for LCSA, VCSA and PCSA are shown in Figure 1 (a, b, c).

\section{Tissue characterization}

Paired tissue characterization data was available for 13 patients. The percent hypoechogenic volume was significantly higher with the $20 \mathrm{MHz}$ system $(96.7 \pm 2.38$ vs. $88.4 \pm 5.53, p<0.0001)$. Figure 2 shows the systematic difference between both systems.

\section{Discussion}

IVUS is currently been employed as a tool to assess atherosclerosis progression/regression in longitudinal studies [6, 15-17]. As the impact of drug therapies on the atherosclerotic plaque burden over time is relatively small, highly reproducible IVUS measurements are essential. A number of IVUS systems are commercially available and the potential impact of inter-catheter variability, in this setting, has not been extensively studied. Mechanical and phased-array catheters have relative advantages and disadvantages. Mechanical catheters have higher resolution but display specific artifacts such as non-uniform rotational distortion. In addition, far field imaging can be more problematic with mechanical catheters due to amplified attenuation and enhanced blood backscatter. On the other hand, phased-array catheters have lower resolution resulting in inferior near-field imaging and as they are not pulled-back within a sheath, are more susceptible to non-uniform pullback speed particularly in tortuous vessels.

Three studies explored the variability between such systems and results were not determinant $[7$, 18, 19].

In an in vitro study conducted by Schoenhagen et al., two mechanical and two phased-array catheters were compared. The largest difference in measurements compared to a phantom model was found with a $30 \mathrm{MHz}$ mechanical catheter [18]. In the study of Hiro et al., the phased array system 

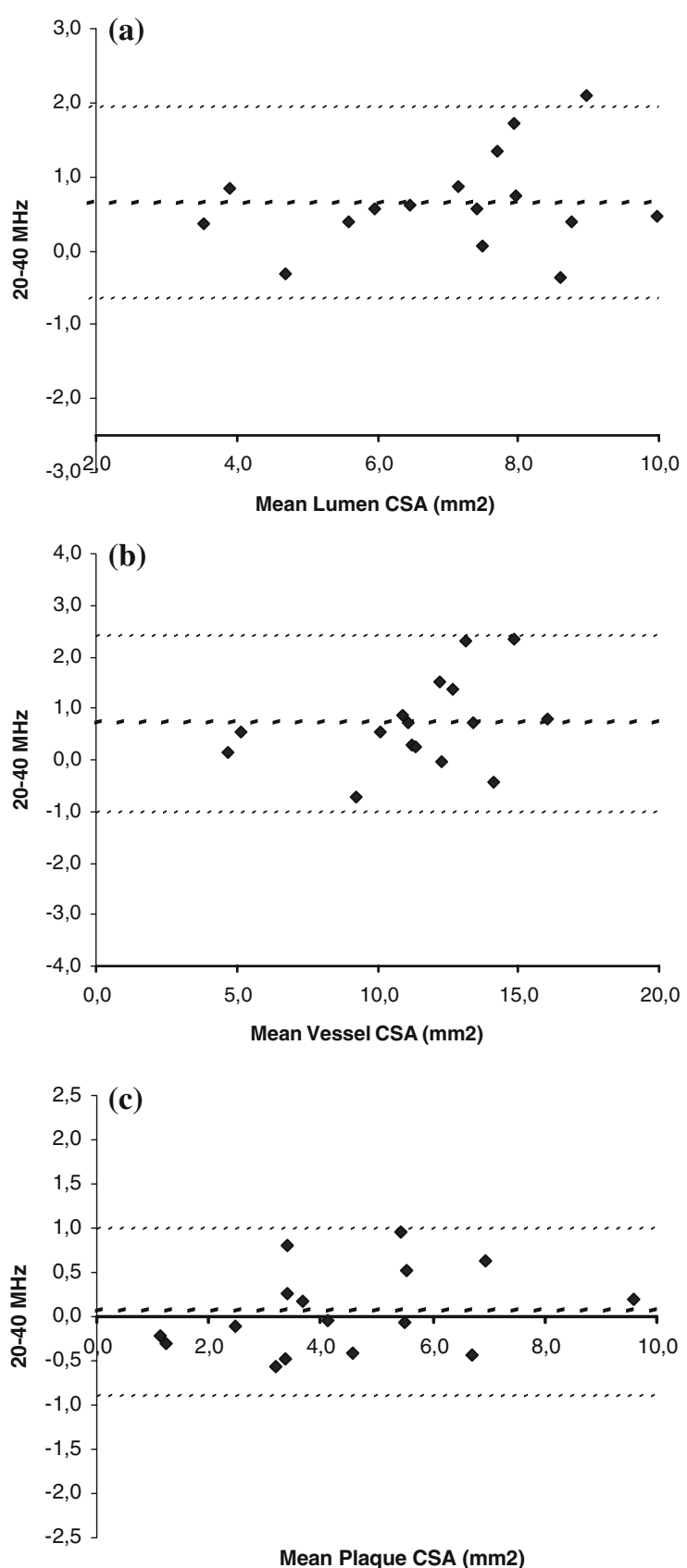

showed a tendency towards a higher correlation with histology in comparison to mechanical systems [8].

The present in vivo study shows a slight systematic difference in lumen and vessel area measurements between the $20 \mathrm{MHz}$ and the $40 \mathrm{MHz}$
Figure 1. (a) Bland-Altman plot where the $X$ axis shows the mean lumen cross sectional area (LCSA, $\mathrm{mm}^{2}$ ), and the $Y$ axis shows the difference between the LCSA measurements by 20 and $40 \mathrm{MHz}$. Thin discontinuous lines show limits of agreement (upper limit $1.95 \mathrm{~mm}^{2}$ and lower limit $-0.65 \mathrm{~mm}^{2}$ ). (b) Bland-Altman plot where the $X$ axis shows the mean vessel cross sectional area (VCSA, $\mathrm{mm}^{2}$ ), and the $Y$ axis shows the difference between the VCSA measurements by 20 and $40 \mathrm{MHz}$. Thin discontinuous lines show limits of agreement (upper limit $2.42 \mathrm{~mm}^{2}$ and lower limit $-1.0 \mathrm{~mm}^{2}$ ). (c) BlandAltman plot where the $X$ axis shows the mean plaque cross sectional area (PCSA, $\mathrm{mm}^{2}$ ), and the $Y$ axis shows the difference between the PCSA measurements by 20 and $40 \mathrm{MHz}$. Thin discontinuous lines show limits of agreement (upper limit $1.0 \mathrm{~mm}^{2}$ and lower limit $-0.88 \mathrm{~mm}^{2}$ ).

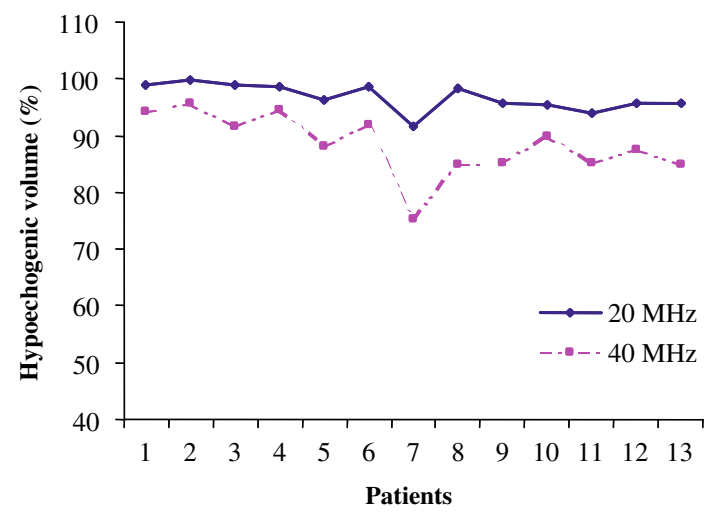

Figure 2. This plot shows the individual (n:13) hypoechogenic volume $(\%)$ for the two systems. The percentage hypoechogenic volume was significantly and systematically higher in the $20 \mathrm{MHz}$ system $(96.7 \pm 2.38$ vs. $88.4 \pm 5.53, p<0.0001)$.

catheters. These results are consistent with previously reported in vivo data [19] . It remains unclear whether such variability is caused by an overestimation of measurements with the phased-array system, or by an underestimation by the mechanical system. It is noteworthy, yet expected, that measurements in vessels with mild disease were subject to greater variability (Figure 1a).

Plaque burden measurements, a key endpoint for atherosclerosis progression/regression trials, showed no difference between the two systems [16]. Similar results have been shown between different mechanical catheters[9]. Notwithstanding, the variability shown in direct measurements, albeit low $(<10 \%)$, is not insignificant when taking into 

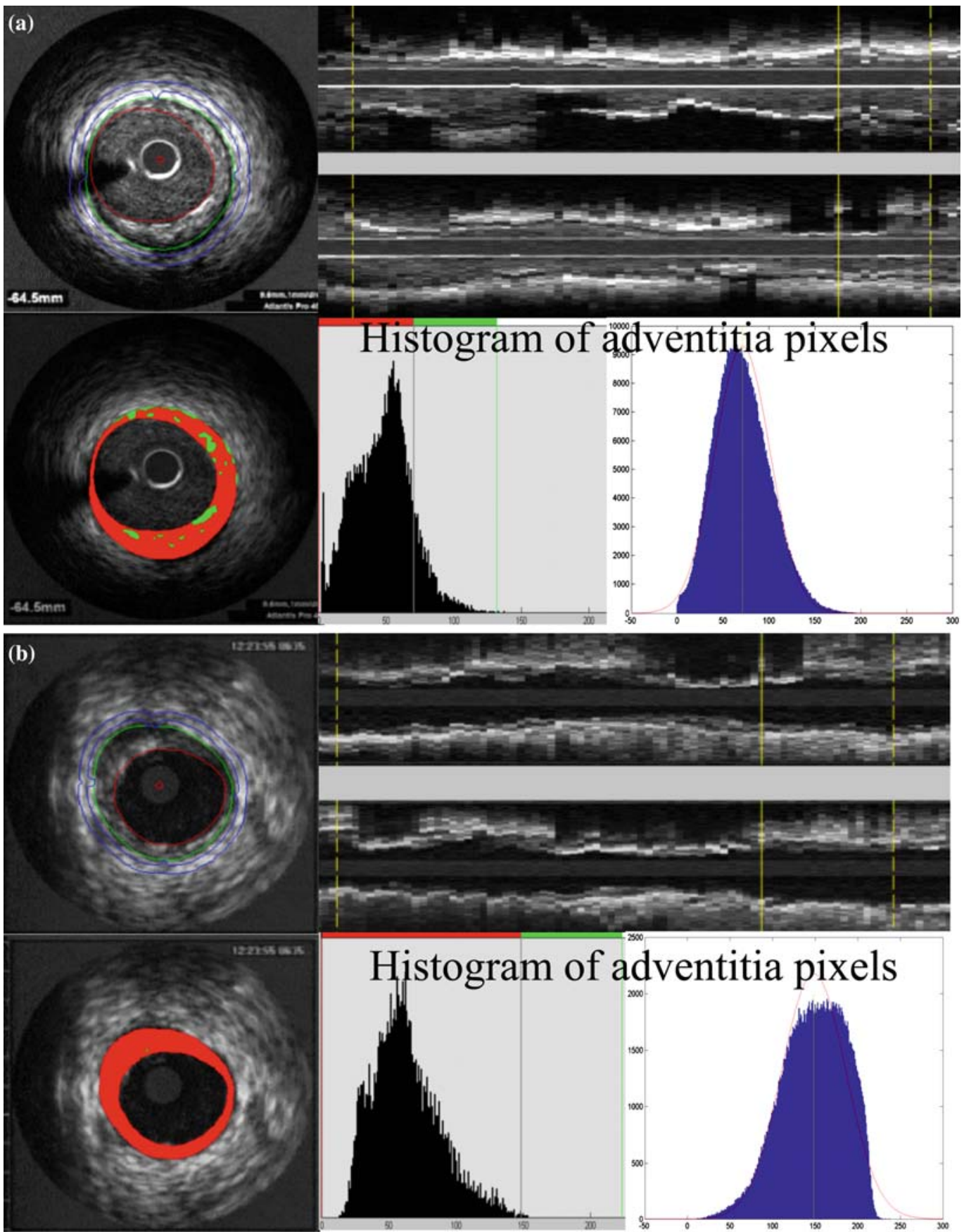

Figure 3. Cross-sectional and longitudinal views of a matched region of interest with 40 (a) and 20 (b) MHz. The adventitia is defined as tissue outside the external elastic membrane. For all non-shadowed adventitia pixels, the mean value and standard deviation are calculated. To observe the suitability, a normal distribution curve based on the same mean and standard deviation histogram is created. Hypoechogenic areas are colored red (dark circle) and hyperechogenic areas green (lighter spots). 
account the relatively small changes observed with drug therapies on plaque burden over time and therefore might contribute to a misinterpretation of their real biological effects.

Our results confirm that the precision required for accurate assessment of modest drug effects could be compromised when different IVUS systems are used in a single study.

Furthermore, the differences shown between catheters are comparable to those previously shown on intra and inter-observer variability [20]. We thus believe that the use of the same IVUS system for longitudinal assessments should be encouraged in order to achieve optimal quality standards [21].

However, the use of a single IVUS system for the conduction of multicenter studies is not easy in practice and it has been previously established that calibration equation methods can correct for differences between catheters.

In line with the morphometric measurements, tissue characterization data with the $20 \mathrm{MHz}$ catheter showed systematically higher hypoechogenic volumes and percentages. It is well known that mechanical catheters have increased acoustic power since they send all the energy in the same direction. Conversely, phased-array catheters send the energy in multiple directions, attenuating their acoustic power. Accordingly, this could potentially be the source for such difference.

\section{Conclusions}

In this in vivo study where we evaluated the agreement between two different catheter designs, plaque burden measurements, a key endpoint for atherosclerosis progression/regression trials, showed no difference between the two systems. However, a significant and systematic variability was detected in direct measurements. Tissue characterization yielded a similar systematic difference between catheters.

It remains unclear whether the difference is caused by an overestimation of measurements with the phased-array system, or by an underestimation by the mechanical system. Nevertheless, until this issue is further explored, we consider that the use of a single IVUS system should be recommended for serial studies.

\section{Limitations}

The number of patients included in this study was small. However, the conductance of large in vivo studies of this type is difficult due to obvious ethical issues. The relatively small amount of plaque in some patients influenced the results as clearly shown in the Bland-Altman plots. Finally, the present study data was processed as analog (video tape). Digital processing could have improved the results. However, we chose the former processing since it is the most commonly used.

\section{References}

1. Nishimura RA, Edwards WD, Warnes CA, et al. Intravascular ultrasound imaging: in vitro validation and pathologic correlation. J Am Coll Cardiol 1990; 16: 145-154.

2. Jaegere Pde, Mudra H, Figulla $\mathrm{H}$, et al. Intravascular ultrasound-guided optimized stent deployment. Immediate and 6 months clinical and angiographic results from the Multicenter Ultrasound Stenting in Coronaries Study (MUSIC Study). Eur Heart J 1998; 19: 1214-1223.

3. Fitzgerald PJ, Oshima A, Hayase M, et al. Final results of the Can Routine Ultrasound Influence Stent Expansion (CRUISE) study. Circulation 2000; 102: 523-530.

4. Rasheed Q, Dhawale PJ, Anderson J, Hodgson JM. Intracoronary ultrasound-defined plaque composition: computer-aided plaque characterization and correlation with histologic samples obtained during directional coronary atherectomy. Am Heart J 1995; 129: 631-637.

5. Nair A, Kuban BD, Tuzcu EM, Schoenhagen P, Nissen SE, Vince DG. Coronary plaque classification with intravascular ultrasound radiofrequency data analysis. Circulation 2002; 106: 2200-2206.

6. Schartl M, Bocksch W, Koschyk DH, et al. Use of intravascular ultrasound to compare effects of different strategies of lipid-lowering therapy on plaque volume and composition in patients with coronary artery disease. Circulation 2001; 104: 387-392.

7. Hiro T, Leung CY, Russo RJ, Karimi H, Farvid AR, Tobis JM. Variability of a three-layered appearance in intravascular ultrasound coronary images: a comparison of morphometric measurements with four intravascular ultrasound systems. Am J Card Imaging 1996; 10: 219-227.

8. Hiro T, Leung CY, Russo RJ, et al. Variability in tissue characterization of atherosclerotic plaque by intravascular ultrasound: a comparison of four intravascular ultrasound systems. Am J Card Imaging 1996; 10: 209-218. 
9. Li Y, Honye J, Saito $\mathrm{S}$, et al. Variability in quantitative measurement of the same segment with two different intravascular ultrasound systems: in vivo and in vitro studies. Catheter Cardiovasc Interv 2004; 62: $175-180$.

10. Nissen SE, Yock P. Intravascular ultrasound: novel pathophysiological insights and current clinical applications. Circulation 2001; 103: 604-616.

11. Prati F, Arbustini E, Labellarte A, et al. Correlation between high frequency intravascular ultrasound and histomorphology in human coronary arteries. Heart 2001; 85: $567-570$.

12. Okimoto T, Imazu M, Hayashi $\mathrm{Y}$, Fujiwara H, Ueda H, Kohno N. Atherosclerotic plaque characterization by quantitative analysis using intravascular ultrasound: correlation with histological and immunohistochemical findings. Circ J 2002; 66: 173-177.

13. Winter SAde, Heller I, Hamers R, et al. Computer assisted three-dimensional plaque characterization in ultracoronary ultrasound studies. Computers in Cardiology 2003; 30: 73-76.

14. Bland JM, Altman DG. Statistical methods for assessing agreement between two methods of clinical measurement. Lancet 1986; 1: 307-310.

15. Nissen SE, Tsunoda T, Tuzcu EM, et al. Effect of recombinant ApoA-I Milano on coronary atherosclerosis in patients with acute coronary syndromes: a randomized controlled trial. Jama 2003; 290: 2292-2300.

16. Nissen SE, Tuzcu EM, Schoenhagen P, et al. Effect of intensive compared with moderate lipid-lowering therapy on progression of coronary atherosclerosis: a randomized controlled trial. Jama 2004; 291: 1071-1080.
17. Jensen LO, Thayssen P, Pedersen KE, Stender S, Haghfelt T. Regression of coronary atherosclerosis by simvastatin: a serial intravascular ultrasound study. Circulation 2004; 110: 265-270.

18. Schoenhagen P, Sapp SK, Tuzcu EM, et al. Variability of area measurements obtained with different intravascular ultrasound catheter systems: Impact on clinical trials and a method for accurate calibration. J Am Soc Echocardiogr 2003; 16: 277-284.

19. Tardif JC, Bertrand OF, Mongrain R, et al. Reliability of mechanical and phased-array designs for serial intravascular ultrasound examinations - animal and clinical studies in stented and non-stented coronary arteries. Int $\mathbf{J}$ Card Imaging 2000; 16: 365-375.

20. Hausmann D, Lundkvist AJ, Friedrich GJ, Mullen WL, Fitzgerald PJ, Yock PG. Intracoronary ultrasound imaging: intraobserver and interobserver variability of morphometric measurements. Am Heart J 1994; 128: 674-680.

21. Von Birgelen C, Hartmann M, Mintz GS, et al. Spectrum of remodeling behavior observed with serial long-term ( $\geq 12$ months) follow-up intravascular ultrasound studies in left main coronary arteries. Am J Cardiol 2004; 93: $1107-1113$.

Address for correspondence: P.W. Serruys, Thoraxcenter, Bd406, Dr. Molewaterplein 40, 3015 GD Rotterdam, The Netherlands

Tel.: + 31-10-4635260; Fax: + 31-10-4369154

E-mail: p.w.j.c.serruys@erasmusmc.nl 